\title{
Research on Direction of Principal Stress Based on Monte-Carlo Method
}

\author{
Li Chen ${ }^{1}$, Yanpeng Feng ${ }^{2}$, Min Xiong ${ }^{1,3}$ and Keqin Ding ${ }^{1, *}$ \\ ${ }^{1}$ China Special Equipment Inspection and Research Institute, Beijing, 100029, China \\ ${ }^{2}$ China Academy of Civil Aviation Science and Technology, Beijing, 100028, China \\ ${ }^{3}$ Wuhan Institute of Technology, Wuhan, 430205, China \\ ${ }^{*}$ Corresponding author
}

\begin{abstract}
Crack initiation and propagation induced by fatigue damage under the action of complex random stress spectrum is the main form of structural failure. The direction of crack initiation is closely related with the maximum principal stress direction, but the maximum principal stress direction of complex random stress spectrum changes with time. Prediction of maximum principal stress direction based on Monte-Carlo method is a feasible method. Firstly, Sampling of random stress spectrum is conducted based on normal distribution, logarithmic normal distribution and Weibull distribution. Then, the MonteCarlo procedures based on three different distributions of prediction of maximum principal stress direction were illustrated. Finally, the engineering application of Monte-Carlo method was carried on the metallurgy crane based on the random stress spectrum data which is obtained from fiber Bragg grating sensor. The results exhibited that the Monte-Carlo method can predicte the crack initiation direction effectively and conveniently.
\end{abstract}

Keywords-component; direction of maximum principle stress; Stress monitoring data; Probabilistic analysis; Monte-Carlo simulation

\section{INTRODUCTION}

Fatigue crack initiation and fatigue crack growth are important damage modes in equipment and structure ${ }^{[1,2]}$. An important example is the large number of longitudinal to web frame connections, which make up an essential part of the plane, ship and crane. The welded structures are exposed to random dynamic loads during the service of equipment and, if not adequately designed, significant fatigue cracking may occur and cause major costs of repair.

A crack starts when the maximum principal stress reaches the tensile strength. The crack direction is in accord with the maximum principal stress direction ${ }^{[3]}$. Although the direction of crack initiation is closely related with the maximum principal stress direction, the maximum principal stress direction of complex random stress spectrum changes with time. Generally speaking, random stress spectrum subjects to normal distribution, logarithmic normal distribution or other distribution $^{[4]}$. Prediction of maximum principal stress direction based on Monte-Carlo method is a feasible method.

Monte-Carlo simulation is a numerical method which involves the use of simulated random numbers to estimate some function of a probability distribution, and is often used to model cases where there is a significant uncertainty, e.g. stress spectrum $^{[5]}$. Monte-Carlo simulation has been used to study fatigue limits ${ }^{[6]}$, polycrystalline microstructural effects ${ }^{[7]}$, stress variation studies on composite single lap ${ }^{[8]}$, and so forth.

The study on maximum principal stress direction under complex random stress spectrum which can more accurately predicte crack initiation and propagation direction is fewer. So in this paper, Monte-Carlo method was adopted to predicte maximum principal stress direction under complex random stress spectrum based on three different distributions.

\section{The CAlculation OF Direction of Principal STRESSES}

The plane stress state is shown in FIGURE I, its stress components $\sigma_{11}, \sigma_{22}$ and $\sigma_{12}$ can be calculated according to the strain monitoring data which is obtained from fiber Bragg grating sensor monitoring based on right angle strain rosette.

As shown in FIGURE I, the directions of maximum (first) and minimum (second) principal stresses are demonstrated as $x_{1}$ and $y_{1}$ direction respectively. The maximum principal direction $x_{1}$ is defined by $\theta_{p}$, and the minimum principal direction $y_{1}$ is perpendicular to the direction $x_{1}$.
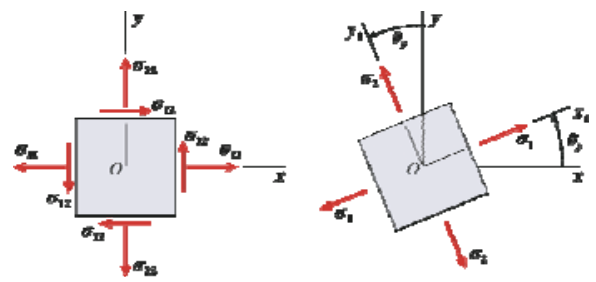

FIGURE I. ILLUSTRATION OF PLANE STRESS STATE AND ITS PRINCIPLE STRESS DIRECTION

The calculation formulas for maximum and minimum principal stresses are as follow:

$$
\sigma_{1,2}=\frac{\left(\sigma_{11}+\sigma_{22}\right)}{2} \pm \sqrt{\left(\frac{\sigma_{11}-\sigma_{22}}{2}\right)^{2}+\sigma_{12}^{2}}
$$

The calculation formulas for $\theta_{p}$ is as follow: 


$$
\cos \left(2 \theta_{p}\right)=\frac{\sigma_{11}-\sigma_{22}}{2 R_{0}} \sin \left(2 \theta_{p}\right)=\frac{\sigma_{12}}{R_{0}}
$$

Where $R_{0}=\sqrt{\left(\frac{\sigma_{11}-\sigma_{22}}{2}\right)^{2}+\sigma_{12}^{2}}$ is always a positive number.

\section{MONTE-CARLO SimUlation}

[1] The plane stress state components were sampled by normal distribution, lognormal distribution and Weibull distribution respectively.

[2] According the formula in second section calculating the probability distribution of the maximal principal stress direction for plane stress state.

[3] Carry out Monte-Carlo simulation no less than 1000 times.

[4] Study on the distribution of mathematical expectation of the first principal stress direction in plane stress state. On the basis of the random stress spectrum, the mathematical expectation can be called the maximal principal stress direction in plane stress state.

\section{A. Monte-Carlo Simulation Based on the Normal Distribution Random Stress Spectrum}

The normal distribution probability density function of three components in plane stress state is shown in formula (3) and FIGURE II.

$$
\left\{\begin{array}{l}
f\left(\sigma_{11}\right)=\frac{1}{\sqrt{2 \pi} s_{1}} \exp \left[-\frac{\left(\sigma_{11}-\mu_{1}\right)^{2}}{2 s_{1}^{2}}\right] \\
f\left(\sigma_{22}\right)=\frac{1}{\sqrt{2 \pi} s_{2}} \exp \left[-\frac{\left(\sigma_{22}-\mu_{2}\right)^{2}}{2 s_{2}^{2}}\right] \\
f\left(\sigma_{12}\right)=\frac{1}{\sqrt{2 \pi} s_{3}} \exp \left[-\frac{\left(\sigma_{12}-\mu_{3}\right)^{2}}{2 s_{3}^{2}}\right]
\end{array}\right.
$$

Where $\mu_{1}=100 \mathrm{Mpa}, \quad s_{1}=12 \mathrm{Mpa}, \quad \mu_{2}=60 \mathrm{Mpa}, \quad s_{2}=8 \mathrm{Mpa}$, $\mu_{3}=100 \mathrm{Mpa}, s_{3}=5 \mathrm{Mpa}$.
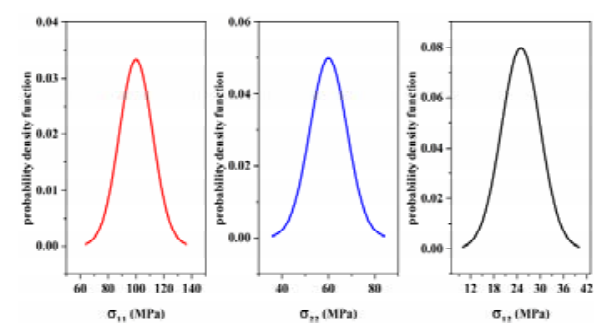

FIGURE II. THE NORMAL DISTRIBUTION PROBABILITY DENSITY FUNCTION
Sampling of stress components based on normal distribution is conducted, and each component extracts 30000 data points. The sampling of $\sigma_{11}$ is shown in FIGURE III, according to the formula (3) for the same sampling of $\sigma_{22}$ and $\sigma_{12}$.

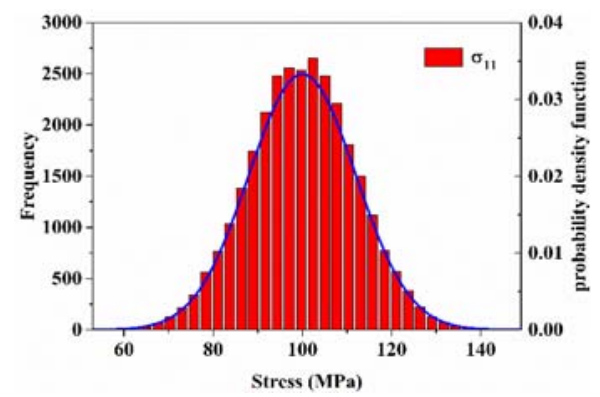

FIGURE III. THE STRESS SAMPLING OF
$\sigma_{11}$ BISTRIBUTION

The three stress components at each moment correspond to a maximum principal stress direction. According to theory in the second section, the 30000 maximum principal stress directions were calculated and the distribution of the maximum principal stress direction is shown in FIGURE IV:

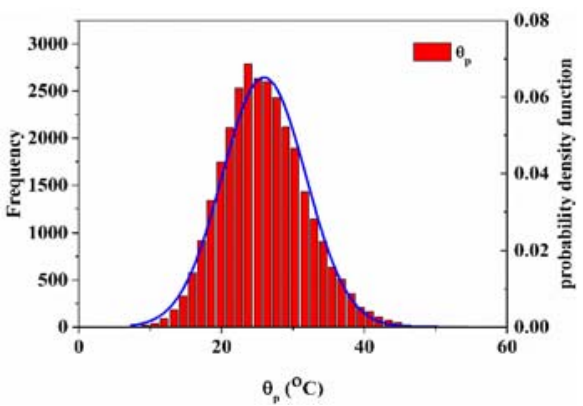

FIGURE IV. DISTRIBUTION OF THE MAXIMUM PRINCIPAL STRESS DIRECTION

As shown in FIGURE IV, the goodness of fit of the curve to the histogram is 0.99 . The results show that the direction of the first principal stress in the plane stress state accords with the normal distribution.

The distribution of the maximum principal stress directions can be obtained from every sampling, calculation and fitting which is accorded with normal distribution. By calculating the mean and standard deviation of the normal distribution, and 1000 groups of mean and standard deviation are obtained by Monte-Carlo simulation, the results are shown in FIGURE V: 


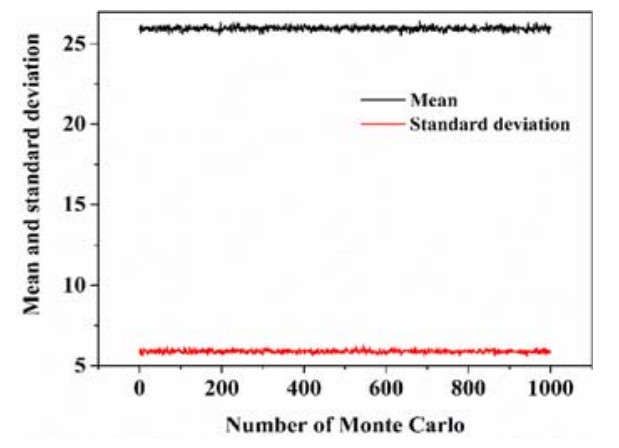

FIGURE V. THE DISTRIBUTIONS OF MEAN AND STANDARD DEVIATION BASED ON 1000 MONTE CARLO SIMULATIONS

The result of Monte-Carlo simulation based on the normal distribution random stress spectrum show that the maximum principal stress direction's mathematical expectation obeys uniform distribution.

\section{B. Monte-Carlo Simulation Based on the Logarithmic Normal Distribution Random Stress Spectrum}

The logarithmic normal distribution probability density function of three components in plane stress state is shown in formula (4) and FIGURE VI.

$$
\left\{\begin{array}{l}
f\left(\sigma_{11}\right)=\frac{1}{\sqrt{2 \pi} s_{1} \sigma_{11}} \exp \left[-\frac{\left(\ln \sigma_{11}-\mu_{1}\right)^{2}}{2 s_{1}^{2}}\right] \\
f\left(\sigma_{22}\right)=\frac{1}{\sqrt{2 \pi} s_{2} \sigma_{22}} \exp \left[-\frac{\left(\ln \sigma_{22}-\mu_{2}\right)^{2}}{2 s_{2}^{2}}\right] \\
f\left(\sigma_{12}\right)=\frac{1}{\sqrt{2 \pi} s_{3} \sigma_{12}} \exp \left[-\frac{\left(\ln \sigma_{12}-\mu_{3}\right)^{2}}{2 s_{3}^{2}}\right]
\end{array}\right.
$$

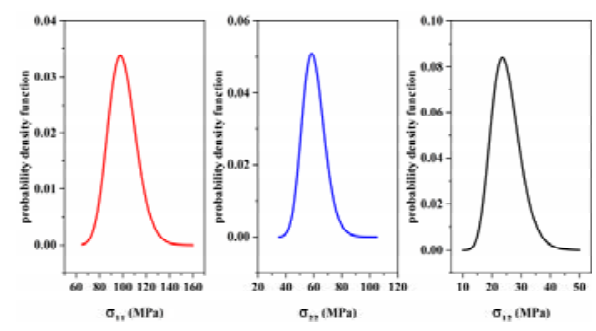

FIGURE VI. THE LOGARITHMIC NORMAL DISTRIBUTION PROBABILITY DENSITY FUNCTION

Sampling of stress components based on logarithmic normal distribution is conducted, and each component extracts 30000 data points. The sampling of $\sigma_{11}$ is shown in FIGURE VII, according to the formula (4) for the same sampling of $\sigma_{22}$ and $\sigma_{12}$.

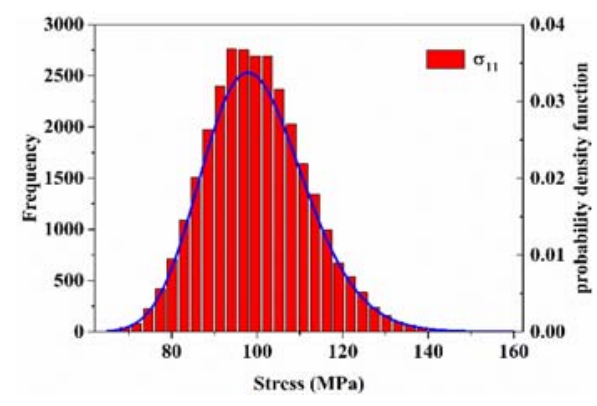

$$
\text { FIGURE VII. THE STRESS SAMPLING OF } \sigma_{11} \text { BASED ON }
$$
LOGARITHMIC NORMAL DISTRIBUTION

The three stress components at each moment correspond to a maximum principal stress direction. According to theory in the second section, the 30000 maximum principal stress directions were calculated and the distribution of the maximum principal stress direction is shown in FIGURE VIII:

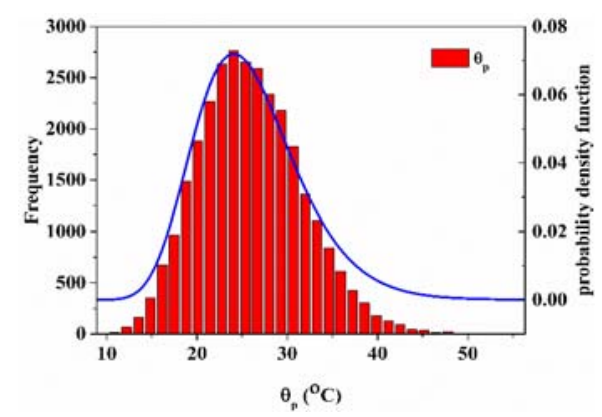

FIGURE VIII. DISTRIBUTION OF THE MAXIMUM PRINCIPAL STRESS DIRECTION

As shown in Figure VIII, the goodness of fit of the curve to the histogram is 0.99 . The results show that the direction of the first principal stress in the plane stress state accords with the logarithmic normal distribution.

The distribution of the maximum principal stress directions can be obtained from every sampling, calculation and fitting which is accorded with logarithmic normal distribution. By calculating the mean and standard deviation of the logarithmic normal distribution, and 1000 groups of mean and standard deviation are obtained by Monte-Carlo simulation, the results are shown in FIGURE IX:

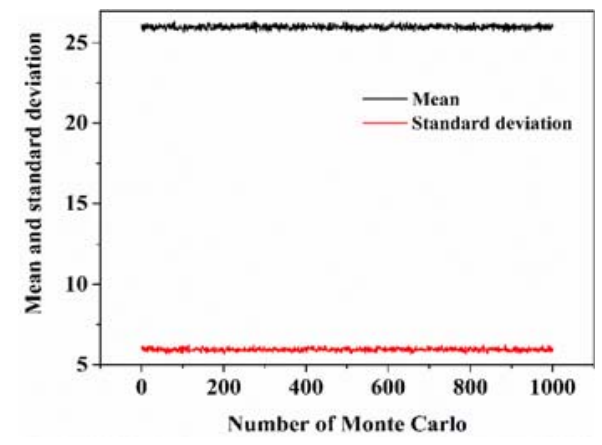

FIGURE IX. THE DISTRIBUTIONS OF MEAN AND STANDARD DEVIATION BASED ON 1000 MONTE CARLO SIMULATIONS 
The result of Monte-Carlo simulation based on the logarithmic normal distribution random stress spectrum show that the maximum principal stress direction's mathematical expectation obeys uniform distribution.

\section{Monte-Carlo Simulation Based on the Weibull Distribution Random Stress Spectrum}

The Weibull distribution probability density function of three components in plane stress state is shown in formula (5) and FIGURE $\mathrm{X}$

$$
\left\{\begin{array}{l}
f\left(\sigma_{11}\right)=\frac{k_{1}}{\lambda_{1}}\left(\frac{\sigma_{11}}{\lambda_{1}}\right)^{k_{1}-1} \exp \left[-\left(\frac{\sigma_{11}}{\lambda_{1}}\right)^{k_{1}}\right] \\
f\left(\sigma_{22}\right)=\frac{k_{2}}{\lambda_{2}}\left(\frac{\sigma_{22}}{\lambda_{2}}\right)^{k_{2}-1} \exp \left[-\left(\frac{\sigma_{22}}{\lambda_{2}}\right)^{k_{2}}\right] \\
f\left(\sigma_{12}\right)=\frac{k_{3}}{\lambda_{3}}\left(\frac{\sigma_{12}}{\lambda_{3}}\right)^{k_{3}-1} \exp \left[-\left(\frac{\sigma_{12}}{\lambda_{3}}\right)^{k_{3}}\right]
\end{array}\right.
$$
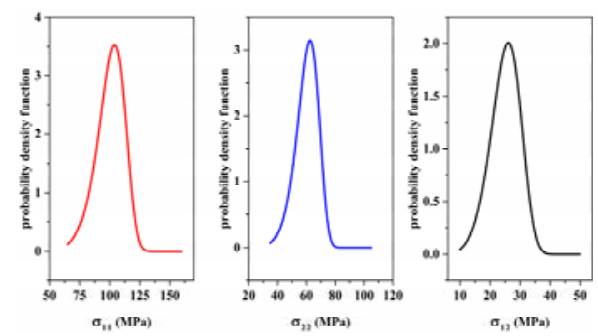

FIGURE X. THE WEIBULL DISTRIBUTION PROBABILITY DENSITY FUNCTION

Sampling of stress components based on Weibull distribution is conducted, and each component extracts 30000 data points. The sampling of $\sigma_{11}$ is shown in FIGURE XI, according to the formula (5) for the same sampling of $\sigma_{22}$ and $\sigma_{12}$.

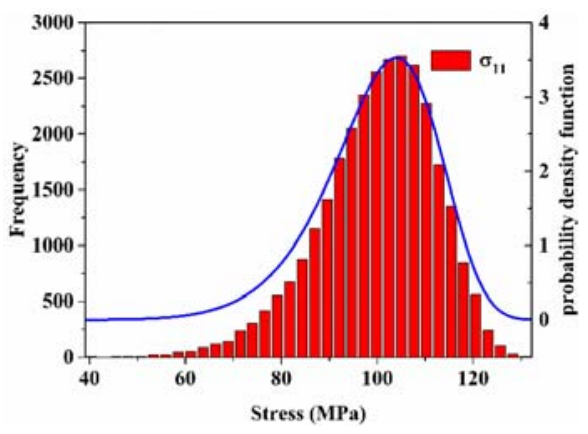

FIGURE XI. THE STRESS SAMPLING OF $\sigma_{11}$ BASED ON WEIBULL DISTRIBUTION
The three stress components at each moment correspond to a maximum principal stress direction. According to theory in the second section, the 30000 maximum principal stress directions were calculated and the distribution of the maximum principal stress direction is shown in Figure XII:

As shown in Figure XII, the goodness of fit of the curve to the histogram is 0.99 . The results show that the direction of the first principal stress in the plane stress state accords with the Weibull distribution.

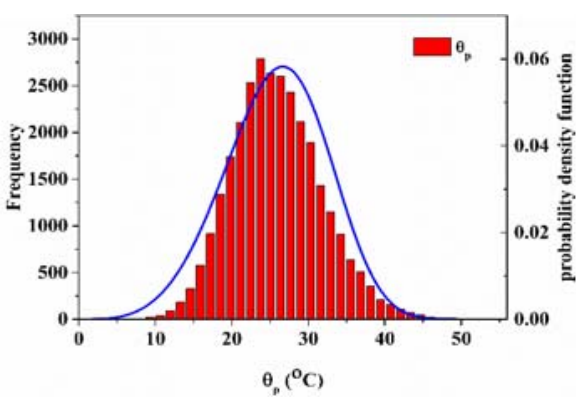

FIGURE XII. DISTRIBUTION OF THE MAXIMUM PRINCIPAL STRESS DIRECTION

The distribution of the maximum principal stress directions can be obtained from every sampling, calculation and fitting which is accorded with Weibull distribution. By calculating the mean and standard deviation of the Weibull distribution, and 1000 groups of mean and standard deviation are obtained by Monte-Carlo simulation, the results are shown in FIGURE XIII:

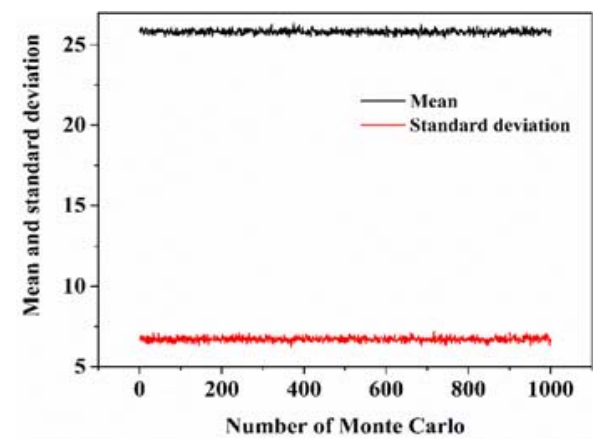

FIGURE XIII. THE DISTRIBUTIONS OF MEAN AND STANDARD DEVIATION BASED ON 1000 MONTE CARLO SIMULATIONS

The result of Monte-Carlo simulation based on the Weibull distribution random stress spectrum show that the maximum principal stress direction's mathematical expectation obeys uniform distribution.

\section{Results and Discussion}

The distribution of maximum principal stress direction is related to sampling probability distribution function. The distribution of maximum principal stress direction of Sampling of stress components based on normal distribution obeys normal distribution, and the same relations for logarithmic normal distribution and Weibull distribution.

The maximum principal stress direction's mathematical expectation which obeys uniform distribution is not related to sampling probability distribution function. 


\section{ENGINEERING APPLICATION}

The position of metallurgy crane plane stress state monitoring based on fiber Bragg grating sensor is shown in FIGURE XIV. The risk of crack initiation on position A2 is highest. So the random stress spectrum on position A2 is studied.

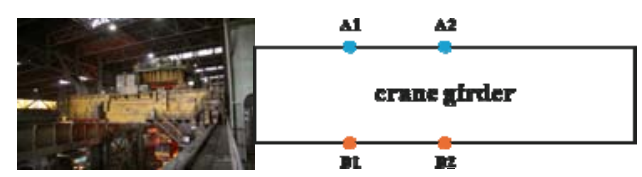

FIGURE XIV. FIBER GRATINGS POSITION OF MONITORING POINT

\section{A. Stress Monitoring Based on Fiber Bragg Grating Sensor}

Main technical advantages of stress monitoring based on fiber Bragg grating sensor are as fellow:

Fiber Bragg grating sensor can be multiplexed so that many sensors lie along a single fiber line. They are compatible with fiber optic data lines, which support huge necessary band width, which in turn support large number of sensors.

Very micro thin. Therefore it can be made compatible with different composite material without changing mechanical properties.

They can withstand high temperatures and pressure and are highly immune to electromagnetic interference.

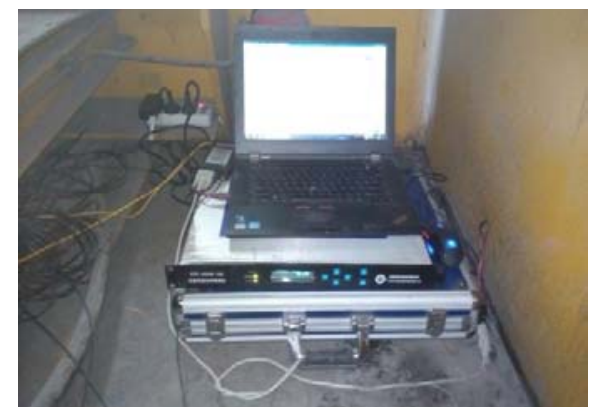

FIGURE XV. EQUIPMENT ADOPTED ON ENGINEERING SITE

\section{B. Monte-Carlo Simulation}

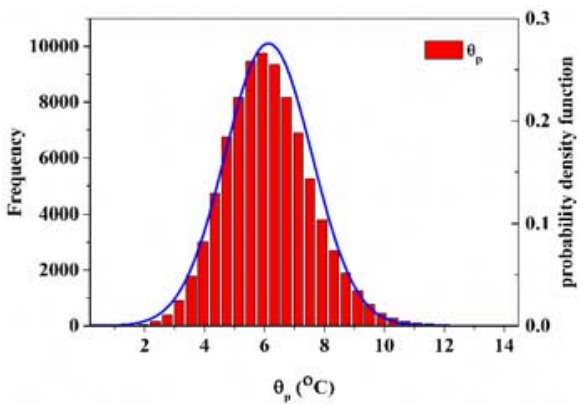

FIGURE XVI. DISTRIBUTION OF THE MAXIMUM PRINCIPAL STRESS DIRECTION IN POSITION A2

Based on the random stress spectrum data which is obtained from fiber Bragg grating sensor, the distribution of the maximum principal stress direction in position A2 of metallurgy crane was given in FIGURE XVI. The mathematical expectation of $\theta_{p}$ in position A2 which also can be seen the crack initiation direction is about $6{ }^{\circ} \mathrm{C}$, and is consistent with the actual situation.

\section{CONCLUSIONS}

Monte-Carlo method was adopted to predicte maximum principal stress direction based on three different distributions which are normal distribution, logarithmic normal distribution and Weibull distribution.

The distribution of maximum principal stress direction is related to sampling probability distribution function. The maximum principal stress direction's mathematical expectation which obeys uniform distribution is not related to sampling probability distribution function. The maximum principal stress direction's mathematical expectation can be seen as the crack initiation direction of the hot region in structure.

The engineering application of Monte-Carlo method was carried on the metallurgy crane based on the random stress spectrum data which is obtained from fiber Bragg grating sensor. The results exhibited that the Monte-Carlo method can predicte the crack initiation direction effectively and conveniently.

\section{ACKNOWLEDGEMENTS}

This work was supported by 863 Program China through 2015AA043702 and by the National Natural Science Foundation of China through grants U1633117.

\section{REFERENCES}

[1] Wang, Y., N.Z. Faruq, L. Susmel. "Evaluation of different techniques in estimating orientation of crack initiation planes and fatigue lifetime under complex multiaxial loading paths". International Journal of Fatigue. vol. 100, Part 2, pp. 521-529, 2017

[2] Yeratapally, S.R., et al. "Bayesian uncertainty quantification and propagation for validation of a microstructure sensitive model for prediction of fatigue crack initiation". Reliability Engineering \& System Safety. vol. 164, pp. 110-123, 2017

[3] Hausslercombe, U. Computational Methods for Reinforced Concrete Structures. in Ix International Conference on Structural Dynamics. 2014.

[4] Xue, G., et al. "Estimation of dynamic stress spectrum distribution in structural fatigue test". Theoretical and Applied Mechanics Letters. vol. 3, pp. 021010, 2013

[5] Xiong, L.H., F. Wang. "Stress Spectrum Analysis on Steel Structure of Gantry Crane". Advanced Materials Research. vol. 291-294, pp. 16341639, 2011

[6] Bai, X., et al. "Measurement and estimation of probabilistic fatigue limits using Monte-Carlo simulations". International Journal of Fatigue. vol. 95, pp. 229-235, 2017

[7] Liu, Y., et al. "Monte Carlo simulation of polycrystalline microstructures and finite element stress analysis". Materials \& Design. vol. 55, pp. 740-746, 2014

[8] Kumar, R.L.V., M.R. Bhat. "Probabilistic stress variation studies on composite single lap joint using Monte Carlo simulation". Composite Structures. vol. 121 , pp. 351-361, 2015 